\title{
The Effect of Early Neuromuscular Electrical Stimulation Therapy in Acute/Subacute Ischemic Stroke Patients With Dysphagia
}

\author{
Kyeong Woo Lee, MD, PhD, Sang Beom Kim, MD, PhD, Jong Hwa Lee, MD, PhD, \\ Sook Joung Lee, MD, PhD, Jae Won Ri, MD, Jin Gee Park, MD
}

Department of Physical Medicine and Rehabilitation, Dong-A University College of Medicine, Busan; Regional Cardiocerebrovascular Center, Dong-A University Hospital, Busan, Korea

Objective To compare the outcome of an early application of neuromuscular electrical stimulation (NMES) combined with traditional dysphagia therapy (TDT) versus traditional dysphagia therapy only in acute/subacute ischemic stroke patients with moderate to severe dysphagia by videofluoroscopic swallowing study (VFSS).

Methods Fifty-seven dysphagic stroke patients were enrolled in a VFSS within 10 days after stroke onset. Patients were randomly assigned into two treatment groups. Thirty-one patients received NMES combined with TDT (NMES/TDT group) and 26 patients received TDT only (TDT group). Electrical stimulation with a maximal tolerable intensity was applied on both suprahyoid muscles for 30 minutes, 5 days per week during 3 weeks. The swallowing function was evaluated at baseline and 3,6, and 12 weeks after baseline. Outcomes of the VFSS were assessed using the Functional Oral Intake Scale (FOIS).

Results The mean ages were 63.5 \pm 11.4 years in the NMES/TDT group and 66.7 \pm 9.5 years in the TDT group. Both groups showed a significant improvement on the FOIS after treatment. The FOIS score was significantly more improved at 3 and 6 weeks after baseline in the NMES/TDT group than in the TDT group $(\mathrm{p}<0.05)$.

Conclusion An early application of NMES combined with TDT showed a positive effect in acute/subacute ischemic stroke patients with dysphagia. These results indicated that the early application of NMES could be used as a supplementary treatment of TDT to help rehabilitate acute/subacute dysphagic stroke patients by improving their swallowing coordination.

Keywords Dysphagia, Acute stroke, Neuromuscular electrical stimulation

Received July 25, 2013; Accepted October 9, 2013

Corresponding author: Jae Won Ri

Department of Physical Medicine and Rehabilitation, Dong-A University Hospital, 26 Daesingongwon-ro, Seo-gu, Busan 602-715, Korea

Tel: +82-51-240-5691, Fax: +82-51-253-8511, E-mail: jun7432@naver.com

(ㄷ) This is an open-access article distributed under the terms of the Creative Commons Attribution Non-Commercial License (http://creativecommons. org/licenses/by-nc/3.0) which permits unrestricted noncommercial use, distribution, and reproduction in any medium, provided the original work is properly cited.

Copyright $\odot 2014$ by Korean Academy of Rehabilitation Medicine

\section{INTRODUCTION}

Dysphagia is extremely common following a stroke and affects $45 \%-65 \%$ of patients suffering from acute stroke. Its incidence is related to lesion size and location $[1,2]$. It is the most significant risk factor for the development of pneumonia and can delay the patient's functional recovery $[2,3]$. Most dysphagia patients recover within 3 weeks of stroke onset, but a recovery is not guaranteed. It may 
take a more protracted course and may lead to serious complications, such as pneumonia, malnutrition or even death [4-6].

A newer treatment modality, neuromuscular electrical stimulation (NMES), provides electrical stimulation on the skin over the throat area. NMES recruits more motor units than volitional contraction and may produce greater gains in muscle strength than exercise alone [7].

Recent studies reported significantly greater improvement for stroke patients in the NMES group compared with other therapeutic methods [8-10]. A previous case control study showed that NMES was a helpful treatment for the feeding of acute/subacute stroke patients with tube dependent dysphagia during inpatient rehabilitation. The previous study enrolled acute hemorrhagic or ischemic stroke patients with brain stem lesion and also severe dysphagic patients who had an initial Functional Oral Intake Scale (FOIS) score of 3 or less [11]. The purpose of this randomized study was to prospectively investigate whether combined NMES and traditional dysphagia therapy (TDT) can improve the swallowing function in acute/subacute supratentorial ischemic stroke patients with moderate to severe dysphagia by VFSS.

\section{MATERIALS AND METHODS}

\section{Method}

Between March 2011 and June 2012, patients were enrolled in this study with a diagnosis of dysphagia by VFSS within 10 days after stroke.

Inclusion criteria were: 1) age between 18 and 80 years; 2) supratentorial ischemic stroke; 3 ) primary diagnosis of stroke confirmed by computed tomography or magnetic resonance imaging; 4) FOIS score of 5 or less; 5) Korean Mini-Mental State Examination (K-MMSE) score of 21 or higher; and 6) stable underlying disease process.

Exclusion criteria for the trial were as follows: 1) presence of dysphagia before stroke; 2) previous stroke history; 3 ) unstable cardiopulmonary status, serious psychological disorder or epilepsy; 4) tumor or radiotherapy of the head and neck region; 5) underwent swallowing therapy before participation in the present study; and 6) unstable medical conditions that may interfere with VFSS.

Ninety-two patients were initially screened; 35 subjects who did not meet the inclusion criteria were excluded as study subjects. Fifty-seven patients met all eligibility criteria. The subjects were randomly assigned into two treatment groups. Thirty-one patients (male 22, female 9) were enrolled from the NMES/TDT group and 26 (male 20, female 6) from the TDT group (Fig. 1). Patients were randomly allocated by a computer generated block randomization sequence into two groups, assigning patients to NMES/TDT group or TDT group with a chance of $50 \%$ to be assigned to therapy.

All patients received TDT including thermal-tactile stimulation with any combination of lingual-strengthening exercises, laryngeal adduction-elevation exercises,

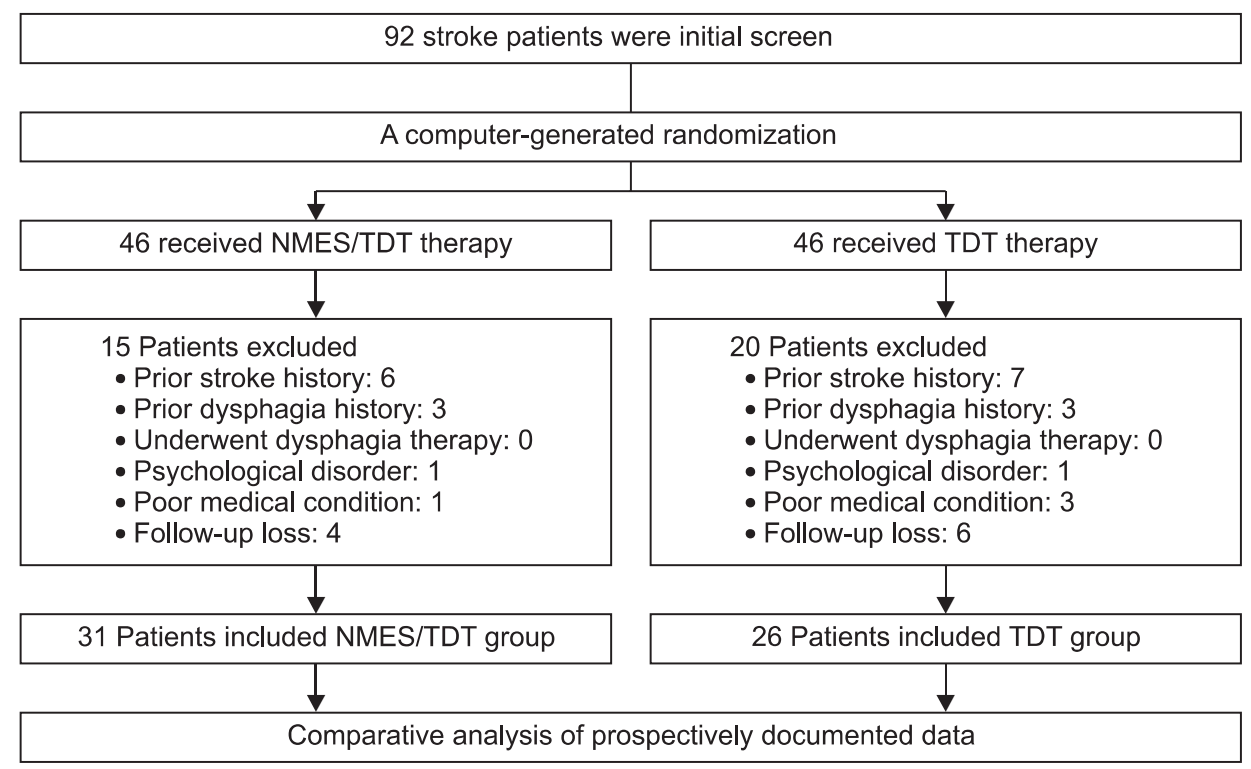

Fig. 1. Flow chart of patient group assignment and treatment. NMES, neuromuscular electrical stimulation; TDT, traditional dysphagia therapy. 
effortful swallow maneuver, Mendelsohn maneuver, Masako maneuver, and Shaker exercises by one speech therapist every day. Thermal-tactile stimulation is a common method that involves stroking or rubbing the anterior faucial pillars with a cold probe prior to having the patient swallow. Both groups received 60 minutes of TDT within a day for 15 days. And NMES (VitalStim Dual Channel Unit and electrodes; Chattanooga Group, Hixson, TN, USA) therapy was applied simultaneously with TDT during the first 30 minutes in the NMES/TDT group. Two sets of electrodes were placed in the infrahyoid area targeting the sternohyoid muscles. NMES intensity was started at $3 \mathrm{~mA}$ and increased gradually by $1 \mathrm{~mA}$. We defined the threshold sensibility when a patient continued to complain about discomfort or pain. Patients measured three times of threshold sensibility and swallowing therapist calculated the mean of the three values. The stimulation intensity was then set at $120 \%$ of the mean threshold value. A pulse rate of $80 \mathrm{~Hz}$ was used with $700 \mu$ s duration. Each NMES session was 30 minutes per day, 5 times a week for a total of 3 weeks.

The results were measured using FOIS (Appendix 1). The FOIS is a 7-point ordinal scale reflecting the dietary intake of patients with dysphagia. It has adequate reliability and validity and has been extensively used in clinical studies of dysphagia to measure the functional oral intake $[7,12]$. We assessed FOIS by VFSS at baseline, after 3,6 , and 12 weeks to compare the degree of improvement between the two groups. All patients were evaluated for dysphagia through VFSS within 10 days (baseline) after stroke and followed-up at 3, 6, and 12 weeks after baseline.

\section{Statistical analysis}

All statistical analyses were performed using the SPSS ver. 18.0K for Windows (SPSS Inc., Chicago, IL, USA). The Friedman test was used to determine changes in patients' swallowing function. The FOIS independent sample t-test validation was used to validate between the two groups. The level of statistical significance was set as p-value less than 0.05 . The Bonferroni method was used as a post-hoc test. Each category was paired and a Wilcoxon signedrank test was performed 6 times; at baseline, 3, 6, and 12 weeks after baseline. The level for statistical significance in the post-hoc test was $0.05 / 6=0.0083$.

\section{RESULTS}

The mean ages of patients were $63.4 \pm 11.4$ years for the NMES/TDT group and $66.7 \pm 9.5$ years for the TDT group. The period from stroke onset to VFSS was $5.5 \pm 2.1$ and $6.3 \pm 2.1$ days. There was no significant difference between both groups. Age, sex distribution, stroke lesion, K-MMSE score, and baseline FOIS were similar in both groups and no significant differences were found (Table 1).

After 15 sessions of NMES with TDT, all patients were continuously applied with TDT. A change in feeding materials of both groups was described in Table 2. Overall, 52 of 57 patients (91.2\%) improved enough to no longer require tubal feeding. However, 2 patients of the NMES/

Table 1. General characteristics of patients

\begin{tabular}{lccc}
\hline \multicolumn{1}{c}{ Characteristic } & NMES/TDT group (n=31) & TDT group (n=26) & p-value \\
\hline Age (yr) & $63.4 \pm 11.4$ & $66.7 \pm 9.5$ & 0.38 \\
\hline Interval from stroke onset to VFSS (day) & $5.5 \pm 2.1$ & $6.3 \pm 2.1$ & 0.26 \\
\hline Interval from stroke onset to treatment (day) & $5.0 \pm 1.4$ & $5.5 \pm 1.1$ & 0.34 \\
Sex (male:female) & $22: 9$ & $20: 6$ & 0.70 \\
lesion location (right:left) & $13: 18$ & $11: 15$ & 0.81 \\
Cortical & $5(16)$ & $4(15)$ & 0.89 \\
Subcortical & $26(84)$ & $22(85)$ & 0.91 \\
K-MMSE & $23.2 \pm 1.5$ & $22.9 \pm 1.4$ & 0.61 \\
Baseline FOIS & $2.0 \pm 1.3$ & $2.2 \pm 1.5$ & 0.49 \\
\hline
\end{tabular}

Values are presented as mean \pm standard deviation or number (\%).

NMES/TDT group, patients treated by neuromuscular electrical stimulation combined with traditional dysphagia therapy; TDT group, patients treated by traditional dysphagia therapy only; K-MMSE: Korean-Mini Mental State Examination; FOIS, Functional Oral Intake Scale. 
Table 2. Comparison of changes in feeding materials after treatment

\begin{tabular}{llccc}
\hline & & Non-oral & Limited diet & Normal diet \\
\hline Baseline & NMES/TDT group & $25(81)$ & $6(19)$ & $0(0)$ \\
& TDT group & $22(85)$ & $4(15)$ & $0(0)$ \\
\hline \multirow{3}{*}{ weeks later } & NMES/TDT group & $12(39)$ & $17(55)$ & $2(6)$ \\
& TDT group & $16(61)$ & $9(35)$ & $1(4)$ \\
\multirow{2}{*}{12 weeks later } & NMES/TDT group & $5(16)$ & $19(61)$ & $7(23)$ \\
& TDT group & $4(15)$ & $18(70)$ & $4(15)$ \\
& NMES/TDT group & $2(7)$ & $14(45)$ & $15(48)$ \\
\hline
\end{tabular}

Values are presented as number (\%).

NMES/TDT group, patients treated by neuromuscular electrical stimulation combined with traditional dysphagia therapy; TDT group, patients treated by traditional dysphagia therapy only.

Table 3. Comparison of mean changes in FOIS after treatment

\begin{tabular}{|cccc}
\hline & $\begin{array}{c}\text { NMES/TDT } \\
\text { group }\end{array}$ & TDT group & p-value \\
\hline Baseline & & & \\
\hline To 3 weeks later & $1.4 \pm 1.0^{\mathrm{a})}$ & $0.5 \pm 0.7^{\mathrm{a})}$ & 0.000 \\
\hline To 6 weeks later & $2.4 \pm 1.3^{\mathrm{b}}{ }^{\mathrm{c}}$ & $1.1 \pm 0.9^{\mathrm{b})}$ & 0.000 \\
\hline To 12 weeks later & $3.1 \pm 1.4^{\mathrm{c}}$ & $1.7 \pm 0.9^{\mathrm{c})}$ & 0.000 \\
\hline 3 weeks & & & \\
\hline To 6 weeks later & $0.9 \pm 0.6^{\mathrm{d})}$ & $0.6 \pm 0.5^{\mathrm{d})}$ & 0.023 \\
\hline To 12 weeks later & $1.7 \pm 1.1^{\mathrm{e})}$ & $1.2 \pm 0.7^{\mathrm{e})}$ & 0.037 \\
\hline 6 weeks & & & \\
\hline To 12 weeks later & $0.7 \pm 1.1^{\mathrm{f})}$ & $0.6 \pm 0.7$ & 0.049 \\
\hline
\end{tabular}

Values are presented as number (\%).

In all periods, NMES/TDT group showed significant improvement compared to TDT group. NMES/TDT group showed improvement of FOIS in all periods. TDT group did not show significant improvement of FOIS in period of 6 weeks to 12 weeks later.

FOIS, Functional Oral Intake Scale; NMES/TDT group, patients treated by neuromuscular electrical stimulation combined with traditional dysphagia therapy; TDT group, patients treated by traditional dysphagia therapy only.

$\mathrm{p}<0.0083$ (independent sample t-test) significant difference ${ }^{\text {a) }}$ between baseline and 3 weeks later in each group, ${ }^{\text {b) be- }}$ tween baseline and 6 weeks later in each group, ${ }^{\mathrm{c})}$ between baseline and 12 weeks later in each group, ${ }^{\text {d) }}$ between 3 weeks and 6 weeks after stroke in each group, ${ }^{\mathrm{e})}$ between 3 weeks and 12 weeks after stroke in each group, ${ }^{\text {f) }}$ between 6 weeks and 12 weeks after stroke in each group.
TDT group and 3 of the TDT group still required tubal feeding for long-term nutrition. The FOIS of the NMES/ TDT group was statistically significant increased in all periods until 12 weeks after treatment (Table 3 ). The TDT group also improved until 12 weeks, but TDT appeared less effective compared with the NMES/TDT group (Fig. 2). The swallowing function of the NMES/TDT group was significantly improved compared with the TDT group in all periods.

Both groups did not show aspiration pneumonia and NMES groups did not show any adverse effects until 12 weeks after stroke.

\section{DISCUSSION}

This study demonstrated that early application of NMES and traditional swallowing rehabilitation showed the potential to improve the FOIS scores and the clinical degree of dysphagia for acute/subacute dysphagic stroke patients. Kushner et al. [11] reported NMES with TDT was a beneficial treatment for acute hemorrhage or ischemic stroke patients with tube dependent severe dysphagia. The measured FOIS score of the supratentorial ischemic stroke patients with moderate to severe dysphagia in our study were differently measured than in the previous study. Additionally, our study was not randomized controlled, but block randomized by a computer generated sequence. NMES is a new treatment for dysphagia, involving the application of electrical current across the skin to excite nerve or muscle tissue during a functional task. NMES can increase the suprahyoid muscle size and improve the range of motion, circulation and muscle en- 


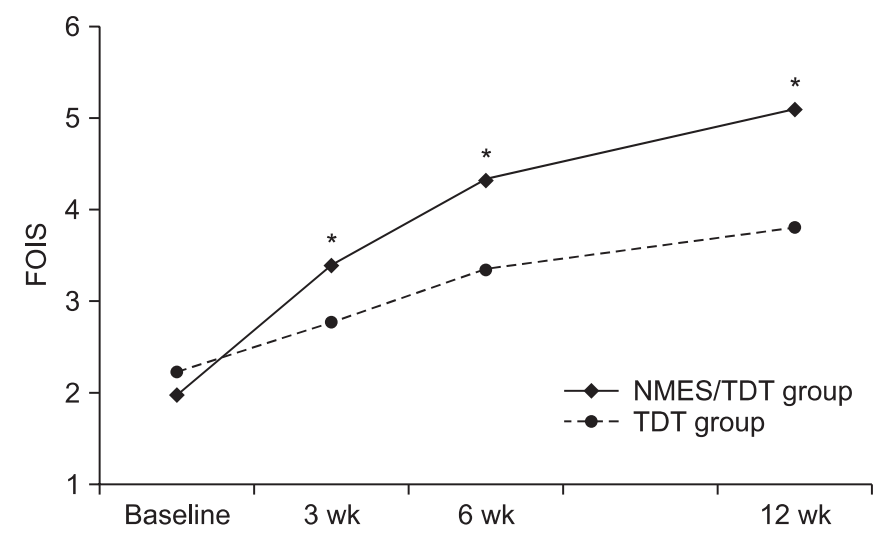

Fig. 2. The change of FOIS in both groups. FOIS significantly increased in NMES/TDT group compare with TDT group at 3 weeks, 6 weeks, and 12 weeks. FOIS, Functional Oral Intake Scale; NMES/TDT group: patients treated by neuromuscular electrical stimulation combined with traditional dysphagia therapy; TDT group, patients treated by traditional dysphagia therapy only. ${ }^{*} \mathrm{p}<0.05$ (independent sample t-test).

durance by increasing the aerobic capacity of the muscle [13]. Recent studies have reported benefits of NMES in the treatment of dysphagia compared with TDT, but most of the studies start dysphagia therapy after 3 weeks of stroke onset [13-18].

Previous investigations have shown that signs of aspiration within the first 3 days of a stroke are associated with a delayed swallowing recovery [19-22]. Recovery from dysphagia after stroke might follow the reorganization of the unaffected motor cortex, but not all patients can recover from dysphagia [23]. Therefore, Odderson et al. [24] showed that an early application of dysphagia therapy for stroke patients was beneficial and cost effective.

Additionally, early NMES application was shown to have more beneficial effect in a dysphagia patient with stroke compared with traditional therapy. Most of the initial tube feeding patients (89.4\%) improved enough to remove the nasogastric tube and maintained their oral feeding with no medical complications thereafter. About $40 \%$ of patients could return to a pre-stroke oral feeding pattern 3 months after the stroke. Our results suggest that early NMES for acute/subacute dysphagic stroke patients can contribute to a rapid and significant improvement in selected patients. However, five patients (NMES/TDT 2 , TDT 3) still required tubal feeding for nutrition at 3-month follow-up. These patients had severe dysphagia at baseline VFSS and did not change at follow-up VFSS. Shaw et al. [25] showed that NMES therapy can improve mild to moderate dysphagia stroke patients, but the most severe dysphagic stroke patients did not gain independence from tubal feeding. Early NMES application with TDT as soon as possible and feeding control guided by VFSS substantially contributed to the improvement of the swallowing function and prevented medical complications, including aspiration pneumonia.

The action mechanisms of NMES have not been fully elucidated yet. NMES is postulated to improve the hyolaryngeal elevation, restore motor function of weak muscles, combat disuse atrophy, enhance sensory awareness and facilitate the muscle contractions [7].

This study has several limitations. First, the study was conducted with a small number of patients. Second, we only measured the functional improvement for a period of 3 to 12 weeks after the stroke. Third, some of the patients had spontaneous recovery, but couldn't be excluded.

In conclusion, we compared the functional swallowing outcomes in acute/subacute stroke patients with an early application of NMES combined with TDT and with TDT only. And as a result, early NMES applied patients showed a significant improvement in their swallowing function at 3, 6, and 12 weeks after onset of stroke compared with the TDT only group. This result suggests that the start of NMES combined with TDT as soon as possible after stroke is a more beneficial treatment for acute/ subacute stroke patients with dysphagia.

\section{CONFLICT OF INTEREST}

No potential conflict of interest relevant to this article was reported.

\section{REFERENCES}

1. Daniels SK, Brailey K, Priestly DH, Herrington LR, Weisberg LA, Foundas AL. Aspiration in patients with acute stroke. Arch Phys Med Rehabil 1998;79:14-9.

2. Roth EJ, Lovell L, Harvey RL, Heinemann AW, Semik P, Diaz S. Incidence of and risk factors for medical complications during stroke rehabilitation. Stroke 2001;32:523-9.

3. Horner J, Massey EW. Silent aspiration following 
stroke. Neurology 1988;38:317-9.

4. Teasell RW, Bach D, McRae M. Prevalence and recovery of aspiration poststroke: a retrospective analysis. Dysphagia 1994;9:35-9.

5. Dziewas R, Ritter M, Schilling M, Konrad C, Oelenberg S, Nabavi DG, et al. Pneumonia in acute stroke patients fed by nasogastric tube. J Neurol Neurosurg Psychiatry 2004;75:852-6.

6. Logemann JA. Update on clinical trials in dysphagia. Dysphagia 2006;21:116-20.

7. Sun SF, Hsu CW, Lin HS, Sun HP, Chang PH, Hsieh $\mathrm{WL}$, et al. Combined neuromuscular electrical stimulation (NMES) with fiberoptic endoscopic evaluation of swallowing (FEES) and traditional swallowing rehabilitation in the treatment of stroke-related dysphagia. Dysphagia 2013;28:557-66.

8. Crary MA, Carnaby-Mann GD, Faunce A. Electrical stimulation therapy for dysphagia: descriptive results of two surveys. Dysphagia 2007;22:165-73.

9. Freed ML, Freed L, Chatburn RL, Christian M. Electrical stimulation for swallowing disorders caused by stroke. Respir Care 2001;46:466-74.

10. Leelamanit V, Limsakul C, Geater A. Synchronized electrical stimulation in treating pharyngeal dysphagia. Laryngoscope 2002;112:2204-10.

11. Kushner DS, Peters K, Eroglu ST, Perless-Carroll M, Johnson-Greene D. Neuromuscular electrical stimulation efficacy in acute stroke feeding tube-dependent dysphagia during inpatient rehabilitation. Am J Phys Med Rehabil 2013;92:486-95.

12. Crary MA, Mann GD, Groher ME. Initial psychometric assessment of a functional oral intake scale for dysphagia in stroke patients. Arch Phys Med Rehabil 2005;86:1516-20.

13. Suiter DM, Leder SB, Ruark JL. Effects of neuromuscular electrical stimulation on submental muscle activity. Dysphagia 2006;21:56-60.

14. Ludlow CL, Humbert I, Saxon K, Poletto C, Sonies $B$, Crujido L. Effects of surface electrical stimulation both at rest and during swallowing in chronic pharyngeal dysphagia. Dysphagia 2007;22:1-10.

15. Kiger M, Brown CS, Watkins L. Dysphagia management: an analysis of patient outcomes using VitalStim therapy compared to traditional swallow therapy. Dysphagia 2006;21:243-53.

16. Gallas S, Marie JP, Leroi AM, Verin E. Sensory transcutaneous electrical stimulation improves post-stroke dysphagic patients. Dysphagia 2010;25:291-7.

17. Blumenfeld L, Hahn Y, Lepage A, Leonard R, Belafsky PC. Transcutaneous electrical stimulation versus traditional dysphagia therapy: a nonconcurrent cohort study. Otolaryngol Head Neck Surg 2006;135:754-7.

18. Verin E, Maltete D, Ouahchi Y, Marie JP, Hannequin D, Massardier EG, et al. Submental sensitive transcutaneous electrical stimulation (SSTES) at home in neurogenic oropharyngeal dysphagia: a pilot study. Ann Phys Rehabil Med 2011;54:366-75.

19. Smithard DG, O'Neill PA, Martin DF, England R. Aspiration following stroke: is it related to the side of the stroke? Clin Rehabil 1997;11:73-6.

20. Schroeder MF, Daniels SK, McClain M, Corey DM, Foundas AL. Clinical and cognitive predictors of swallowing recovery in stroke. J Rehabil Res Dev 2006;43:301-10.

21. Kumar S, Langmore S, Goddeau RP Jr, Alhazzani A, Selim M, Caplan LR, et al. Predictors of percutaneous endoscopic gastrostomy tube placement in patients with severe dysphagia from an acute-subacute hemispheric infarction. J Stroke Cerebrovasc Dis 2012;21:114-20.

22. Ickenstein GW, Hohlig C, Prosiegel M, Koch H, Dziewas R, Bodechtel U, et al. Prediction of outcome in neurogenic oropharyngeal dysphagia within 72 hours of acute stroke. J Stroke Cerebrovasc Dis 2012;21:569-76.

23. Singh S, Hamdy S. Dysphagia in stroke patients. Postgrad Med J 2006;82:383-91.

24. Odderson IR, Keaton JC, McKenna BS. Swallow management in patients on an acute stroke pathway: quality is cost effective. Arch Phys Med Rehabil 1995;76:11303.

25. Shaw GY, Sechtem PR, Searl J, Keller K, Rawi TA, Dowdy E. Transcutaneous neuromuscular electrical stimulation (VitalStim) curative therapy for severe dysphagia: myth or reality? Ann Otol Rhinol Laryngol 2007;116:36-44. 
Appendix 1. Functional Oral Intake Scale

\begin{tabular}{clll}
\hline Score & Performance & \multicolumn{1}{c}{ Implication } & Deficit \\
\hline 1 & Aspirates saliva & Nothing by mouth & Profound \\
\hline 2 & Tube dependent & Nothing by mouth/minimal trials & Profound \\
3 & Tube dependent & Full trials by mouth & Severe \\
4 & Total oral & Single texture trials & Moderate \\
\hline 5 & Total oral & Multiple texture trials & Mild \\
6 & Total oral & By mouth/restrictions & Minimal \\
\hline 7 & Regular diet & By mouth/no restrictions & Normal \\
\hline
\end{tabular}

